\title{
Intercalibration between HIRS/2 and HIRS/3 channel 12 based on physical considerations
}

\section{Klaus Gierens et al.}

Correspondence to: Klaus Gierens (klaus.gierens@dlr.de)

The copyright of individual parts of the supplement might differ from the CC BY 3.0 License. 


\section{Remarks}

In this supplement we show actual weighting functions for a number of profiles recorded in Lindenberg and Sodankylä und we provide a bit more information on the notion and use of weighting functions (kernels) and Jacobians.

\section{Actual weighting functions}

In Figure S1 we show actual weighting functions for profiles from Lindenberg and Sodankylä. The dates of the radiosonde launches are given in the figures. Two radiative transfer simulations have been performed for each profile, one for HIRS channel 12 on NOAA 14 (solid curves) and one for the same channel on NOAA 15 (curves with circles). It is seen that the NOAA 15-curves peak at higher altitudes than the NOAA 14-curves.

Note that libradtran does not currently provide a mechanism to compute these weighting profiles. Jacobians are provided neither. The curves have been computed by extracting and processing the relevant quantities from "verbose" output.

\section{On the notion and use of weighting functions and Jacobians}

It seems useful to provide some thoughts on the notions weighting function, weighting kernel, and Jacobian.

The solution of the radiative transfer equation in a simple setting is

$$
I=I_{0} \mathcal{T}(0)+\int_{0}^{\infty} B(z) \frac{d \mathcal{T}(z)}{d z} d z=: I_{0} \mathcal{T}(0)+\int_{0}^{\infty} B(z) W(z) d z,
$$

that is, the weighting function is $W=d \mathcal{T} / d z$ (Harries, 1997). Here, $B$ is the Planck function, $\mathcal{T}$ is transmission, $z$ is altitude and $z=0$ refers to the ground. If the quantity of interest is the radiant intensity $I$, or equivalently, the brightness temperature, then $W$ is the weighing function (or weighting kernel) that we need. This is the function, for which we use a generic form in the manuscript and which we plotted in the first reply (note that $d \mathcal{T} / d z=\chi \mathcal{T}$, where $\chi$ is the extinction coefficient).

Jackson and Bates (2001) use the same type of solution of the RT equation (without the surface term), using pressure or log pressure as the vertical coordinate. But they call $d \mathcal{T} / d \ln p$ simply the transmission function. In their paper the word "weighting" is reserved for something different. They use the retrieval formula by Soden and Bretherton (1993), in inverse form:

$$
a+b T_{12}=\ln \left(\frac{\langle R H\rangle P_{0}}{\beta \cos \theta}\right) .
$$

This equation contains two averages, $\langle R H\rangle$, and $\beta=\langle d \ln T / d \ln p\rangle$. Jackson and Bates study how different formulations of these averages using two different weighting functions affect the scatter of the results. One of these weighting functions is given as fixed weights from their table 
1, the other is, according to a suggestion in Stephens et al. 1996, the transmission weighting function from above, that is $d \mathcal{T} / d \ln p$.

Neither Harries nor Jackson and Bates use Jacobians. The latter are derivatives of the brightness temperature to any factor influencing it, in our case it would be $d T_{12} / d R H(z)$. Of course this can also be considered as a kind of weighting function, but to our view it would more properly be considered a sensitivity profile or function of influence.

The interpretation (i.e. meaning) of $W(z)$ differs from that of the Jacobian. As said, $d T_{12} / d R H(z)$ gives the change of brightness temperature for a change of $\mathrm{RH}$ in a certain altitude, while $W(z)$ measures the contribution of photons emitted at $z$ to the signal that reaches the satellite. Eventually, $\mathcal{T}(z)$ is a function of $R H(z)$ (more correctly, a functional: at each $z, \mathcal{T}(z)$ is a function of the complete $\mathrm{RH}$ profile from $z$ to the top of the atmosphere). Thus, the weighting function $W(z)$, since it depends on $\mathrm{RH}$ and other quantities (temperature, gas concentrations, ...) is more general than Jacobians. Both quantities are useful, but probably to different degrees in different applications.

For individual profiles $\mathcal{T}(z)$ and $R H(z)$, the information contained in individual weighting functions and Jacobians is confined to the respective profile. While we know an expression for a generic weighting profile, we don't know one for a generic Jacobian, and in fact we doubt that one could be derived. Thus it is necessary and justified for our argumentation to use generic weighing functions.

\section{References}

Harries, J.E.: Atmospheric radiation and atmospheric humidity. Q.J.R. Meteorol. Soc., 123, 2171-2186, 1997.

Jackson, D. and Bates, J.: Upper tropospheric humidity algorithm assessment, JGR, 106, 32 259-32 270, 2001.

Soden, B. and Bretherton, F.: Upper tropospheric relative humidity from the GOES $6.7 \mu \mathrm{m}$ channel: Method and climatology for July 1987, J. Geophys. Res., 98, 16 669-16 688, 1993.

Stephens, G., Jackson, D., and Wittmeyer, I.: Global observations of upper-tropospheric water vapor derived from TOVS radiance data, J. Climate, 9, 305-326, 1996. 

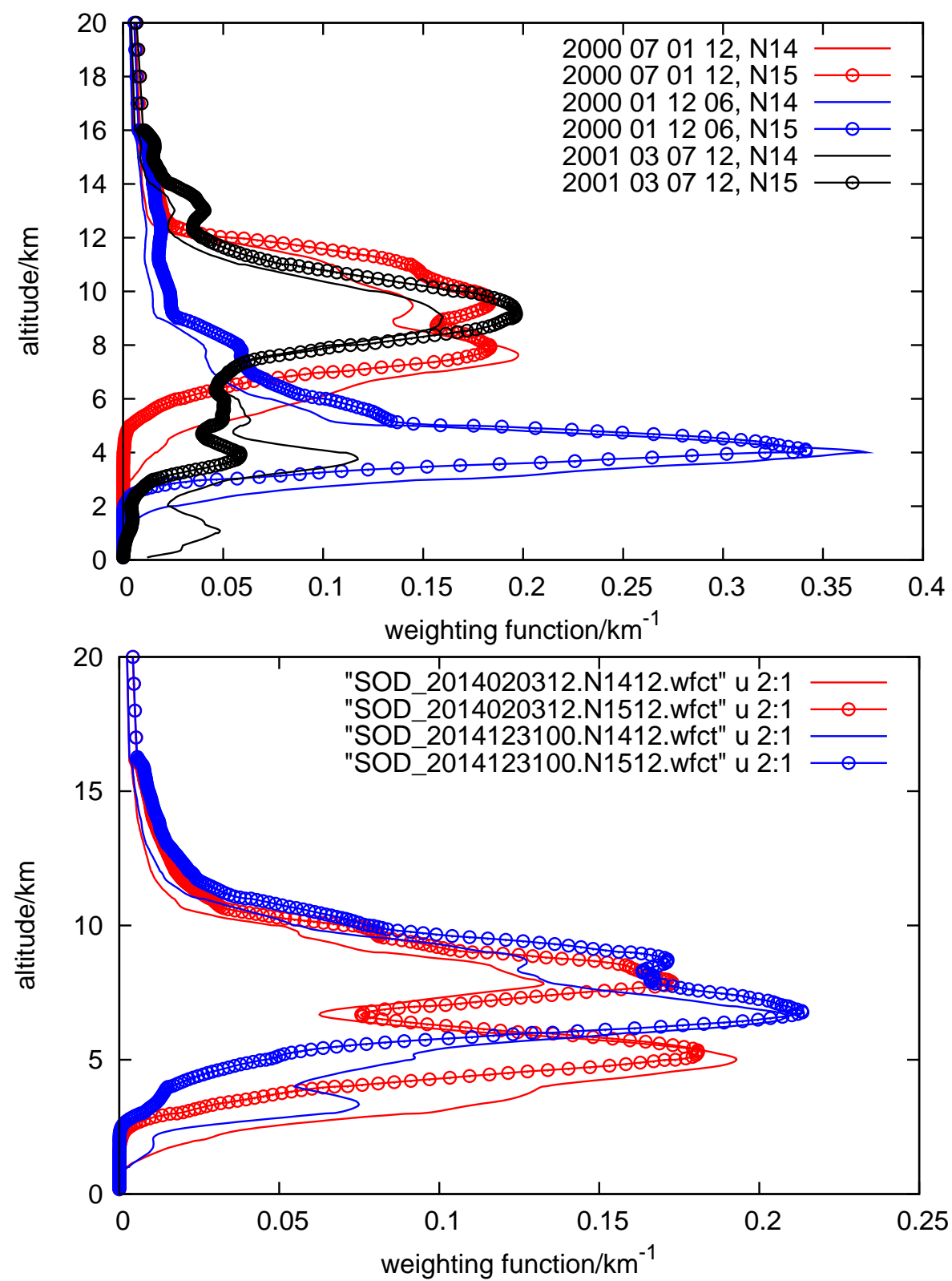

Figure S1: Top: Actual channel 12 weighting functions for NOAA 14 (simple lines) and NOAA 15 (lines with circles) for three radiosonde profiles from Lindenberg (dates given). Bottom: the same for Sodankylä. 